\title{
Mixed Cryoglobulinemic Vasculitis: A Case Report
}

\author{
Akio Hirama ${ }^{1}$, Akiko Mii ${ }^{1}$, Yusuke Arakawa ${ }^{1}$, Toshiki Funakoshi ${ }^{1}$, \\ Mita Ko ${ }^{1}$, Yukinao Sakai ${ }^{1}$, Akira Shimizu ${ }^{2}$ and Shuichi Tsuruoka ${ }^{1}$ \\ ${ }^{1}$ Department of Nephrology, Nippon Medical School, Tokyo, Japan \\ ${ }^{2}$ Department of Pathology, Nippon Medical School, Tokyo, Japan
}

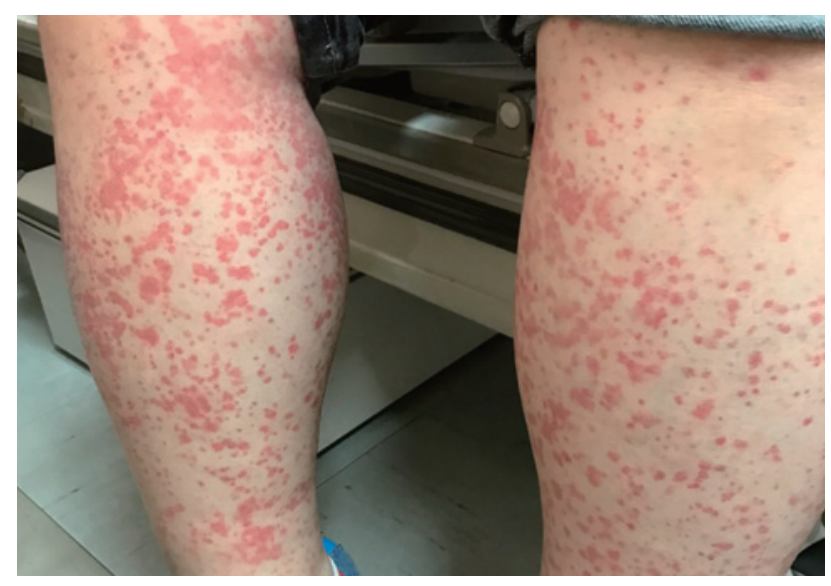

Fig. 1

\section{Introduction}

Cryoglobulins are immunoglobulins that precipitate in vitro below $37^{\circ} \mathrm{C}$ and can cause multi-organ damage ${ }^{1}$ Cryoglobulinemia is associated with infection, autoimmune disease, and hematologic malignancy. The pathogenesis of tissue injury mainly follows two mechanisms, cryoglobulin precipitation in the microcirculation and immune-complex-mediated inflammation of blood vessels.

\section{Case Presentation}

A 50-year-old woman was referred to our hospital because of proteinuria, microscopic hematuria, and rapidly progressing renal insufficiency. The patient had edema of the lower extremities and purpura (Fig. 1). Arthralgia and weakness were not found. Laboratory data indicated renal insufficiency, anemia, hypergammaglobulinemia, and hypocomplementemia. Rheumatoid factor and cryoglobulin tests were also positive. However, no evidence of infection or systemic disease was found. Renal biopsy results revealed membranoproliferative and endocapillary proliferative lesions (Fig. 2). The formation of cellular and fibrocellular crescents were also sporadically seen. Immunofluorescence showed granular staining for polyclonal immunoglobulins and complements in the mesangial and subendothelial areas (Fig. 2). Electron-dense deposits with subtle fibrillary structures could be observed on electron microscopy (Fig. 3). Based on the above-mentioned findings, we diagnosed the patient with essential mixed cryoglobulinemic vasculitis.

Conflict of Interest: The authors declare that they have no competing interests.

Correspondence to Akiko Mii, MD, PhD, Department of Nephrology, Nippon Medical School, 1-1-5 Sendagi, Bunkyo-ku, Tokyo 113-8602, Japan 


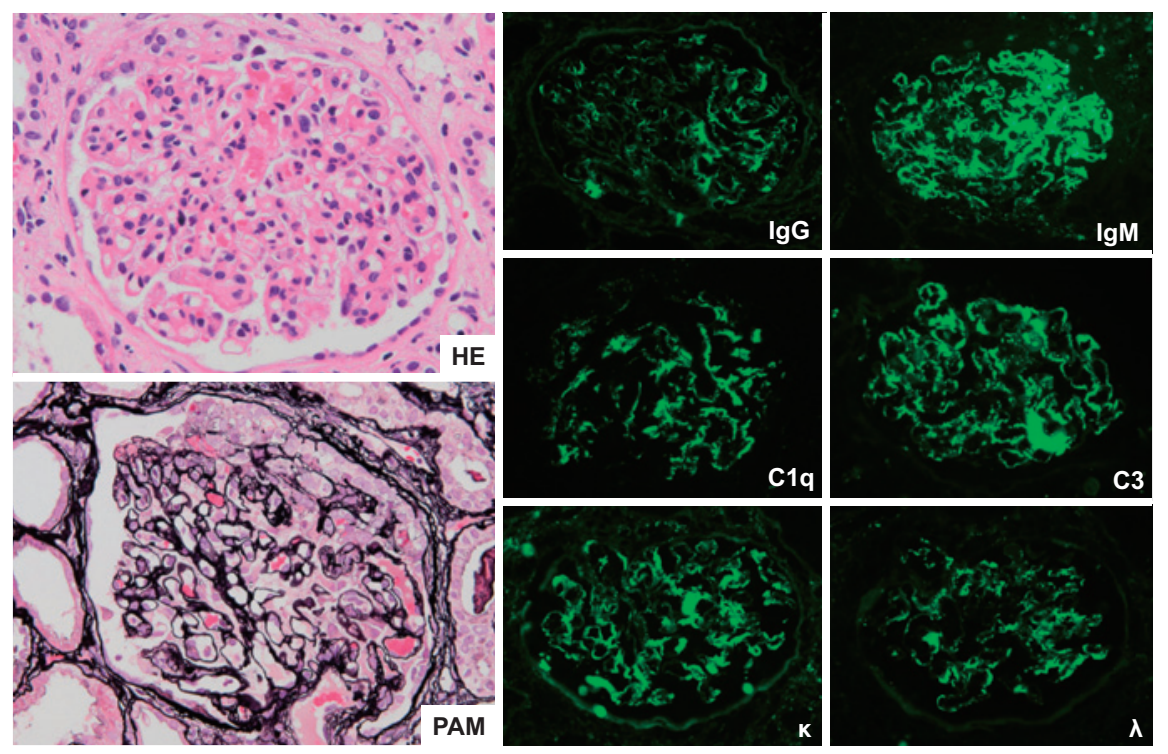

Fig. 2

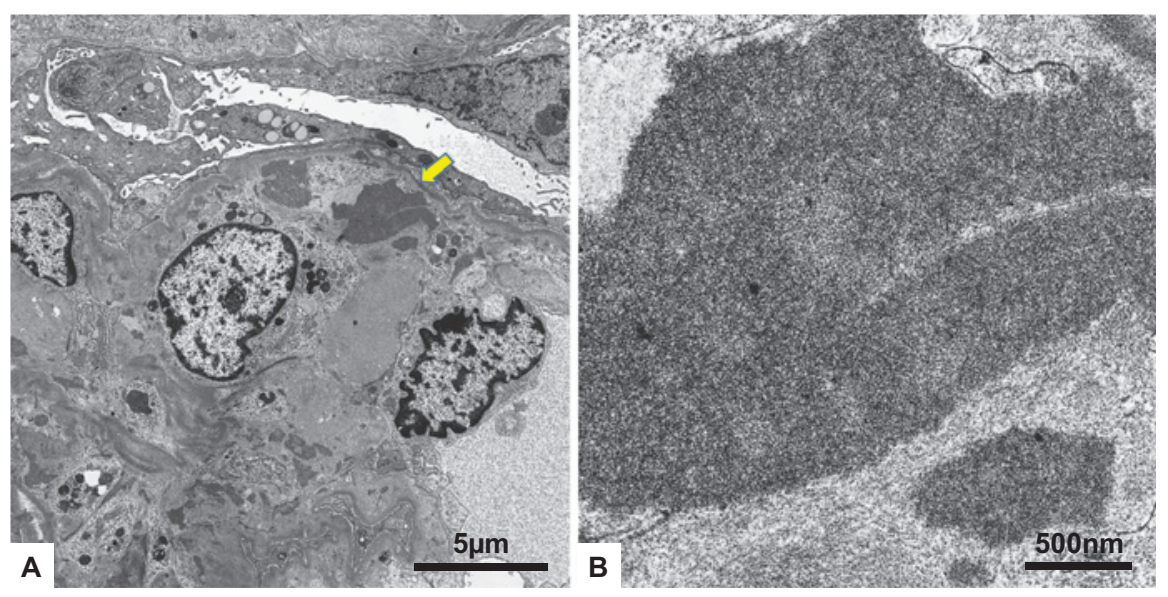

Fig. 3

Fig. 1 Cutaneous purpura occurs with high frequency in cryoglobulinemic vasculitis. Many small petechial lesions can be observed on the legs.

Fig. 2 The light microscopy image shows membranoproliferative and endocapillary proliferative lesions with inflammatory cell infiltration in the glomeruli. The formation of a fibrocellular crescent can be observed. The immunofluorescence study result shows granular staining of polyclonal immunoglobulins (IgG and $\mathrm{IgM}$ ) and complements (C1q and C3) in mesangial and subendothelial areas (original magnification $\times 400$ ).

Fig. 3 The electron microscopy image shows glomerular subendothelial deposits and podocyte foot process effacement. The arrow indicates organized deposits in the subendothelial areas (A). The highmagnification image of the deposits shows a subtle fibrillary appearance rather than a microtubular structure (B).

\section{Reference}

1. Ramos-Casals M, Stone JH, Cid MC, Bosch X: The cryoglobulinaemias. Lancet 2012; 379: 348-360. 